\title{
Food-burying behavior in red imported fire ants (Hymenoptera: Formicidae)
}

\author{
Wenquan Qin ${ }^{1}$, Xuan Chen ${ }^{2}$, Linda M Hooper-Bùi ${ }^{3}$, Jiacheng Cai ${ }^{4}$, Lei Wang ${ }^{5}$, Zhaohui Sun ${ }^{1}$, Xiujun Wen \\ 1 , Cai Wang Corresp. 1 \\ ${ }^{1}$ Guangdong Key Laboratory for Innovation Development and Utilization of Forest Plant Germplasm, College of Forestry and Landscape Architecture, \\ South China Agricultural University, Guangzhou, Guangdong, China \\ 2 Department of Biology, Salisbury University, Salisbury, MD, USA \\ 3 Department of Environmental Sciences, Louisiana State University, Baton Rouge, LA, USA \\ 4 Department of Mathematics and Computer Science, Salisbury University, Salisbury, MD, USA \\ ${ }^{5}$ College of Agriculture, South China Agricultural University, Guangzhou, Guangdong, China \\ Corresponding Author: Cai Wang \\ Email address: wangcai@scau.edu.cn
}

The food-burying behavior has been reported in many mammals and birds, but was rarely observed in invertebrates. The red imported fire ants, Solenopsis invicta Buren, is an invasive pest in many areas of the world that usually performing food burying during the foraging processes. However, the impacted factors and measureable patterns of this behavior is largely unknown. In the present study, food-burying versus food-transport behaviors of $S$. invicta were observed under laboratory and field conditions. When starved (no food was provided for 37 days) in the laboratory, food (sausage) was consumed by large numbers of ants, and few burying behaviors were observed. However, when food was provided until satiation of the colonies, food-transport was suppressed and significantly more soil particles were relocated on the food and graph paper square (where the food was placed) when compared with these colonies exposed to starved conditions. Videotapes showed that soil particles $\left(1.47 \pm 0.09 \mathrm{~mm}^{2}\right)$ were preferentially placed adjacent to (in contact with) the food items at thebeginning; and after the edges were covered, ants transported significantly smaller soil particles $\left(1.13 \pm 0.06 \mathrm{~mm}^{2}\right)$ to cover the food. Meanwhile, larger particles $\left(1.96 \pm 0.08 \mathrm{~mm}^{2}\right)$ were pulled/dragged around (but not in contact with) the food. Interestingly, only a small number of ants, mainly the small workers, were involved in food burying, and the ants tended to repeatedly transport soil particles. Twelve patterns of particle transport were identified, and soil particles were most frequently picked from the foraging arena and subsequently placed adjacent to the food. In the field, almost all released food was actively transported by S. invicta workers, and no burying behavior was observed. Our results show that the food-burying behavior of $S$. invicta may be associated with the suppressed foraging activity, and the burying task may be carried out by certain groups of workers. 
1 Food-Burying Behavior in Red Imported Fire Ants (Hymenoptera:

2 Formicidae)

3

4 Wenquan Qin ${ }^{1}$, Xuan Chen ${ }^{2}$, Linda M. Hooper-Bùi ${ }^{3}$, Jiacheng Cai ${ }^{4}$, Lei Wang ${ }^{5}$, Zhaohui Sun ${ }^{1}$,

5 Xiujun Wen ${ }^{1, *}$, Cai Wang ${ }^{1, *}$

7 Affiliations

$8 \quad{ }^{1}$ Guangdong Key Laboratory for Innovation Development and Utilization of Forest Plant

9 Germplasm, College of Forestry and Landscape Architecture, South China Agricultural

10 University, Guangzhou, China. ${ }^{2}$ Department of Biology, Salisbury University, Salisbury, MD,

11 USA. ${ }^{3}$ Department of Environmental Sciences, Louisiana State University, Baton Rouge, LA,

12 USA. ${ }^{4}$ Department of Mathematics and Computer Science, Salisbury University, Salisbury, MD,

13 USA. ${ }^{5}$ College of Agriculture, South China Agricultural University, Guangzhou, China

15 * Corresponding Authors: Xiujun Wen, Cai Wang

Email address: wenxiujun@scau.edu.cn,wangcai@scau.edu.cn 
Abstract: The food-burying behavior has been reported in many mammals and birds, but was rarely observed in invertebrates. The red imported fire ants, Solenopsis invicta Buren, is an invasive pest in many areas of the world that usually performing food burying during the foraging processes. However, the impacted factors and measureable patterns of this behavior is largely unknown. In the present study, food-burying versus food-transport behaviors of S. invicta were observed under laboratory and field conditions. When starved (no food was provided for 37 days) in the laboratory, food (sausage) was consumed by large numbers of ants, and few burying behaviors were observed. However, when food was provided until satiation of the colonies, foodtransport was suppressed and significantly more soil particles were relocated on the food and 
INTRODUCTION

The red imported fire ant, Solenopsis invicta Buren, is a significant pest that has been introduced into many areas around the world, including North America, Australia, China, and Asian-Pacific regions (Morrison et al., 2004; Zeng et al., 2005; Ascunce et al., 2011; Wetterer, 2013; Wylie \& Janssen-May, 2017). The invasion of S. invicta has represented a major threat to native arthropods and small vertebrates in many ecological communities (Holway et al., 2002; Orrock \& Danielson, 2004; Thawley \& Langkilde, 2016; Darracq et al., 2017). Baiting is one of the most successful methods for the control of S. invicta, especially when area-wide fire ant management is needed (Williams, Collins \& Oi, 2001; Rust \& Su, 2012). For example, after three years of broadcast bait treatments, Wylie et al. (2016) reported that S. invicta has been eradicated in two infested areas of Queensland, Australia. The effectiveness of baiting largely depends on the foraging behaviors of S. invicta (i.e. food/bait searching, recruitment, feeding, transportation, and other behaviors associated with foraging), which have been widely studied in the past decades (e.g. Cook et al., 2010; Wilder et al., 2011; Tschinkel, 2011; Wang et al., 2016; 2018).

However, some aspects of foraging behaviors have not yet been thoroughly investigated. One example is the utilization of particles (soil and debris) by S. invicta for different purposes during foraging. For example, Barber et al. (1989) found that when honey was provided, $S$. invicta workers covered honey with particles which were then carried back to the nest and sucked like a sponge. Wang et al. (2018) observed that when S. invicta workers fed upon a droplet of sugar water, the ants usually placed soil particles into the edge of the droplet to break 
60 the water tension. Then they were able to suck the sucrose water that eventually spread on the

61 soil particles. Similar particle-utilization behaviors were also observed during the liquid-feeding

62 processes of ants Pogonomyrmex badius (Latreille) (Morrill, 1972), Aphaenogaster subterranea

63 Latreille, and Aphaenogaster senilis Mayr (Maák et al., 2017).

64 Besides, $S$. invicta performs some unique particle-utilization behaviors that have not been

65 reported in other ant species. For example, S. invicta workers sometimes carry soil or other

66 coarse particles to "bury" large food items that cannot be transported immediately (Hölldobler \&

67 Wilson, 1990; Xu et al., 2007). The food-burying behaviors have been extensively studied in

68 some mammals and birds. Those animals bury food items when the food is not needed at the

69 moment, or when the environment does not allow the individuals to consume the food

70 immediately (Abbott \& Quink, 1970; Miyaki, 1987; Hayashida, 1989; Wauters, Tosi \& Gurnell,

71 2002; Su, Ma \& Zong, 2007; Zong et al., 2010; 2014; Steele et al., 2011). However, to our best

72 knowledge, no previous studies have focused on the food burying behavior in an invertebrate,

73 eusocial species. The food-burying behavior may reduce the effective of fire ant baiting because

74 the granular baits (containing soybean oil as the attractant) may deteriorate within a short period

75 of time under natural conditions if they are buried rather than transported by ants.

76 Our preliminary observations showed that the burying and foraging behaviors seem

77 "competitive" because food items (e.g. frozen crickets and sausage) usually were either

78 transported by groups of $S$. invicta workers or buried (partially or entirely) with soil particles. In

79 the latter case, the food was usually not consumed by ants evidently (WQ and CW, unpublished

80 data). Based on these observations, we hypothesized that S. invicta would forage food when ants 
were starved, and perform burying behavior when plenty food was provided. In the present study, the foraging and burying behaviors were first compared when ants were starved or fed until satiation in the laboratory, and then observed under field conditions.

Division of labors (different groups of workers are specialized to carry out certain tasks) during food/soil transport have been previously described in some ants (Beshers \& Fewell, 2001). For example, both of the nest excavation (upward transport of excavated sand) and food storage (downward transport of seed) processes of the harvester ant, Pogonomyrmex badius (Latreille), were carried out in stages by different groups of individuals (Tschinkel, Rink \& kwapich, 2015). However, it is unclear whether S. invicta workers show division of labors and measurable behavioral patterns when they transport soil particles to bury the food on ground. To verify this, we took videos in the laboratory to determine the behaviors of individual ants involved with the food-burying process.

\section{MATERIALS AND METHODS}

\section{Ant Collection and Rearing}

Eight colonies of $S$. invicta were collected from different ant mounds ( $>5 \mathrm{~m}$ from each other) in the greenbelt near the South China Agricultural University $\left(23^{\circ} 9^{\prime} \mathrm{N}, 113^{\circ} 21^{\prime} \mathrm{E}\right)$, Guangzhou, China, on 12 November 2016. The methods provided by Wang et al. (2018) were modified to collect and maintain S. invicta colonies. In brief, ant mounds that contained large number of eggs, larvae, pupae and adults were rapidly transferred by shovel to a $1.5 \mathrm{~L}$ plastic box $(16 \times 12 \times 9.5 \mathrm{~cm}$ $[\mathrm{L} \times \mathrm{W} \times \mathrm{H}])$ which was sealed and brought to the laboratory within $1 \mathrm{~h}$. A foraging arena 
102 (uncovered plastic storage container, $29 \times 16 \times 9.5 \mathrm{~cm}[\mathrm{~L} \times \mathrm{W} \times \mathrm{H}]$ ) was prepared by coating the

103 walls with Teflon (Dupont, Beijing, China) to prevent ant escape. The bottom of the foraging

104 arenas was evenly covered with 200 g loamy clay soil (44\% sand, $21 \%$ silt and 35\% clay). Here

105 we decided to use the loamy clay soil because it is in red color and therefore can be easily

106 distinct from ants which have dark-colored bodies. Also, we previously observed that S. invicta

107 relocated particles of loamy clay to bury food items in the field. Before use, soil was ground

108 using wooden mortars and pestles, and sifted through a 3-mm sieve to remove any coarse

109

materials (our preliminary studies showed that S. invicta workers did not transport soil particles >

$3 \mathrm{~mm}$ in diameter). The sizes of sifted particles used in the present study were ranging from 0 to

$11113.7 \mathrm{~mm}^{2}$. A silicon tube $(0.4 \mathrm{~cm}$ in inner diameter, and $20 \mathrm{~cm}$ in length) was used to connect the

112 nest box (containing nest materials) and foraging arena (Fig. 1a). Ant colonies were allowed to

113 acclimate in the laboratory for $\sim 2$ months (Fig. 1b). Eppendorf tubes (10 mL) containing the $20 \%$

114 honey/water solution and plain water were placed in the foraging arenas, and frozen crickets were provided ad libitum.

\section{Food-Burying versus Food-Transport Activities under Starved or Fed Conditions}

118 The procedures of the laboratory study were shown on Fig. 1b. We stopped feeding ants with crickets and honey/water solution 37 days before the experiment, but plain water was still provided. O’Brien and Hooper-Bùi (2005) reported that $4 \mathrm{~d}$ of starvation of $S$. invicta colonies are needed when they were reared under laboratory conditions to simulate foraging ants in the 
123

124

125

126

127

test was conducted when ants were completely starved. Temperature was maintained at $21 \pm 2{ }^{\circ} \mathrm{C}$ during this and following experiments. Two pieces of graph paper $(5 \times 5 \mathrm{~cm}$, with 10 lines per centimeter) coated with a layer of plastic membrane were placed on half of the foraging arena that was far from the entrance of the nesting box (Fig. 1a). A small piece $(10 \times 10 \times 1.5 \mathrm{~mm})$ of sausage (Guangdong Shuanghui Food Co., Ltd., Qingyuan, Guangdong, China) or a false food (square acrylic plates $[10 \times 10 \times 1.5 \mathrm{~mm}]$ covered with a layer of plastic membrane with the same color as the sausage) was glued on the center of the graph paper. We used the false food as the control to investigate whether a non-food object (that bears a similar search image to food items) can also trigger foraging/burying behaviors of $S$. invicta. A high-resolution picture was taken for each graph paper every 60 minutes for four hours, and the number of foraging ants that on or adjacent to (in contact with) the food items (real or false) were counted after $1 \mathrm{~h}$ of food release.

At the end of the experiment, soil particles on the graph paper and food were collected with soft forceps and weighed using a $0.1 \mathrm{mg}$ electronic balance. Although some particles were transported around (no contact with) the food, they were still considered to be the result of burying behavior (see results). This test was repeated 32 times (four times for each colony). Only one test was conducted for each colony each day. After the first test, ants were again fed $20 \%$ honey/water solution and frozen crickets. The second test was conducted after ants were fed until satiation for $15 \mathrm{~d}$ (Fig. 1b), and the procedures were the same to the first test as mentioned above.

For each test, the active foraging behavior was defined as $\geq 20 \%$ of the food was consumed by ants, and the active burying behavior was defined as $\geq 10$ soil particles were found on or adjacent to (in contact with) food items (real or false). The number of foraging ants and weight 
144 of particles was compared using two-way analysis of variance (ANOVA, SAS 9.4, SAS Institute,

145 Cary, NC) with ant colony as the random effect and treatments (combinations of food types [real

146 or false] and feeding status [starved or fed]) as the fixed effect, followed by Tukey's Honest

147 Significant Differences (HSD) tests for multiple comparisons. The significance level was 148 determined at $\alpha=0.05$ for all tests.

\section{Behavioral Patterns of Individual Ants under Fed Conditions}

151

152

153

154

155

156

157

158

159

160

161

162

163

164

Some colonies always exhibited active burying behavior after ants were fed until satiation (see

results), thus two of them were selected for videotaping to determine the behavioral patterns of individual ants. Only real food was videotaped because false food triggered little burying activity (see results). A piece of graph paper was placed in the foraging arena, and a small piece of sausage was affixed on the center of the graph paper as described above. Video was then taken for the following $2 \mathrm{~h}$. Only one video was taken for each colony each day. In total, 10 videos (five videos for each colony) were obtained, and we assume that these data are independent. A screenshot was taken at 15 min intervals during the video, and the number of soil particles found on or adjacent to, or around the food was counted. At each time interval, the number of particles - recorded only when $\geq 5$ particles were placed on the food and graph paper on each location was converted to percentage, which was then transformed to $\ln$-ratios because of the sum constraint of the compositional data (Aitchison, 1986; Kucera \& Malmgren, 1998). The transformed data were compared using repeated measures ANOVA (SPSS 24, SPSS Inc., Chicago, IL) with time as the within-subjects effect and location as the between-subjects effect. 
165 The degrees of freedom were corrected with Greenhouse-Geisser method due to the violation of 166 Mauchly's Test of Sphericity (Greenhouse \& Geisser, 1959). One-way ANOVA was performed 167 for each time interval for multiple comparisons. At the end of each video $(2 \mathrm{~h})$, the size of each particle was measured using the ImageJ software (US National Institutes of Health, Bethesda, USA), and compared among locations using the one-way ANOVA (SAS 9.4). food for dissecting or sucking), or wandering on the graph paper was counted every 5 min, and compared using repeated measures ANOVA (SPSS 24) with time as the within-subjects effect and behavior as the between-subjects effect. The degrees of freedom were adjusted with Greenhouse-Geisser method due to the violation of Mauchly's Test of Sphericity. One-way ANOVA was performed for each time interval for multiple comparisons. Ants involved in particle transport were traced and the following information was recorded: (i) worker-size class of each particle transporter (small, medium, or large worker); (ii) time spent by each ant during transport; (iii) number of relocated particles for each ant; and (iv) average time spent transporting each particle by each ant. The time and number (measurement ii-iv) were compared among ant sizes (large, medium and small workers) using the one-way ANOVA (SAS 9.4). The pattern of each transport event was classified based on the location where the soil particle was picked and placed on the graph paper. The time between picking (or when transporting onto the graph paper from the foraging arena) and placing of each particle was recorded and compared among transport patterns using the one-way ANOVA (SAS 9.4). After each ANOVA, Tukey's HSD test was conducted for post-hoc analyses. 


\section{Field Observation}

188 The field investigation was performed in June and July 2017, near the SCAU where the S. invicta activity was previously detected. Forty-four study sites (with $>5 \mathrm{~m}$ interval between any adjacent sites) were randomly selected along the green belt. The tests were conducted from 17:20 to $19: 20$ (the temperature was between $30^{\circ} \mathrm{C}$ and $32^{\circ} \mathrm{C}$, the relative humidity was between $70 \%$ and $80 \%$, and study sites were not under direct sunlight during the study). Right before the test, a square $(4.8 \times 4.8 \mathrm{~cm})$ of the $\mathrm{V}$-tech ${ }^{\circledR}$ tape (Guangzhou, China) was affixed on the ground (either on soil or on the cement roadbed), and a piece of sausage $(10 \times 10 \times 2 \mathrm{~mm})$ was fixed on the center of the tape using an insect pin. A photograph was taken at the 60 min mark to determine the behavioral activities (food-burying versus food-transport) of ants. Similar to the laboratory study, the active burying behavior was determined if there were $\geq 10$ particles found on or adjacent to the food. The foraging behaviors were also recorded if numerous ants $(>30)$ were recruited to dissect the sausage, or if the food was transported away from the tape by ants.

\section{RESULTS}

\section{Burying versus Foraging Activities under Starved or Fed Conditions}

Sausage (real food) attracted a large number of ants from colonies under starved conditions (Fig.

2). At $4 \mathrm{~h}$, most sausage was actively foraged by starved ants, whereas little was consumed by

fed ant colonies (Fig. 3a). The number of foraging ants on or adjacent to (in contact with) the 
$207565.01 ; \mathrm{df}=3,96 ; P<0.0001 ;$ Fig. 3b). False food attracted few ants throughout the test whether 208 the ants were starved or fed (Fig. 2 and 3b).

A high frequency of burying behavior was observed by ants from fed colonies (Fig. 3c).

Significantly more soil particles (measured in weight) were transported by fed ants compared with starved ants $(F=36.94 ; \mathrm{df}=3,96 ; P<0.0001$; Fig. $3 \mathrm{~d})$. Interestingly, five ant colonies always showed the active burying behavior when they were fed, whereas the other colonies never exhibited similar behavior (Supplementary Table S1). False food triggered very few burying activities regardless whether the colonies were starved or fed (Fig. 3c, d).

\section{Behavioral Patterns of Individual Ants under Fed Conditions}

For the percentage (transformed data) of soil particles, there was no significant effect from the

locations $(F=2.59, \mathrm{df}=2,15, P=0.108)$ or time $(F=0.00, \mathrm{df}=2.7,40.8, P=1.000)$, while the

interaction effect between time and location was significant $(F=11.73, \mathrm{df}=5.4,40.8, P<0.001)$.

At $15 \mathrm{~min}$, ant placed significantly more particles adjacent to the food than that on or around the

food (Fig. 4a, b; statistical results are shown in Supplementary Table S2). From 30-60 min, significantly more particles were adjacent to the food than on the food, but both were not significantly different from the percentage of particles around the food (Fig. 4a, b). From 75-90 min, similar percentages of particles were found on, adjacent to, or around the food (Fig. 4b).

From 105-120 min, significantly more particles were around the food than on the food, but both were not significantly different from the percentage of particles adjacent to the food (Fig. 4a, b).

In total, 620 particles were found in the three locations at the end of the experiment. The mean 
228 size of soil particles on the food $\left(1.13 \pm 0.06 \mathrm{~mm}^{2}[\right.$ mean $\left.\pm \mathrm{SE}, \mathrm{n}=165]\right)$ was significantly

229 smaller than those particles adjacent to the food $\left(1.47 \pm 0.09 \mathrm{~mm}^{2}[\mathrm{mean} \pm \mathrm{SE}, \mathrm{n}=148]\right)$. In turn,

230 both these sets of particles were significantly smaller than those around the food $(1.96 \pm 0.08$

$231 \mathrm{~mm}^{2}[$ mean $\left.\pm \mathrm{SE}, \mathrm{n}=307]\right)(F=26.56 ; \mathrm{df}=2,617 ; P<0.0001 ;$ Fig. 4c $)$.

232 There were significant effects from ant behavior $(F=122.23, \mathrm{df}=2,27, P<0.001)$, time $(F$

$233=3.80, \mathrm{df}=7.2,193.3, P=0.001)$, and their interaction $(F=4.21, \mathrm{df}=14.3,193.3, P<0.001)$.

234 Only a few ants were involved in burying, which was significantly fewer than ants that wandered

235 on the graph paper (during the 2-h mark), or foraged on the food (from 25-30 and 55-120 min)

236 (Fig. 5a; statistical results are shown in Supplementary Table S3). In total, 133 soil transporters

237 were tracked during videotaping. Most of them were small workers (Fig. 5b). On average, each

238 soil transporter repeatedly moved $6.7 \pm 0.8($ mean \pm SE) particles, which lasting for $381.4 \pm 48.0$

239 (mean $\pm \mathrm{SE}$ ) seconds. The time spent by each ant during transport and number of relocated

240 particle were not significantly different among small, medium, and large workers (time: $F=1.94$;

$241 \mathrm{df}=2,130 ; P=0.1480$; number: $F=1.52 ; \mathrm{df}=2,130 ; P=0.2229 ;$ Figs. 5c, d). Also, the

242 average time spent transporting each particle was similar when they were transported by workers

243 of different sizes $(F=1.04 ; \mathrm{df}=2,130 ; P=0.3578$; Fig. 5e $)$.

244 Moreover, 12 patterns were identified based on 881 particle transport events, including the

245 long-distance ( 3 patterns) as well as the short-distance (9 patterns) transport (Fig. 6a). Particles

246 were most frequently picked from the foraging arena and subsequently placed adjacent to the

247 food (Fig. 6b). Interestingly, significantly more time was required when particles were picked 
248 adjacent to the food and relocated on the food compared with many other patterns $(F=7.25 ; \mathrm{df}=$

249 11, 869; $P<0.0001$; Fig. 6c).

250

\section{Field Observation}

252

253

254

255

256

257

258

259

260

261

262

263

264

265

266

267

268

The active foraging behaviors were observed in almost all tests under field conditions.

Meanwhile, soil particles and other coarse materials were found around (not in contact with) the food. However, no food was directly buried by particles (Fig. 7).

\section{DISCUSSION}

The burying behaviors have been previously reported in some vertebrates such as mammals and birds. For example, Frehner et al. (2017) observed that the American badgers (Taxidea taxus [Schreber]) partially or entirely buried the carcasses of juvenile domestic cow (Bos Taurus L.) that was much larger than the badgers themselves. Also, many seed-eating rodent (e.g. Peromyscus leucopus [Rafinesque], Clethrionomys gapperi [Vigors], Sciurus vulgaris L., etc.) and birds (e.g. Nucifraga caryocatactes Brehm, Sciurus spp., etc.) usually hoard the seeds by burying them below the leaf litter or soil (Abbott \& Quink, 1970; Miyaki, 1987; Hayashida, 1989;

Wauters, Tosi \& Gurnell, 2002; Su, Ma \& Zong, 2007; Zong et al., 2010; 2014; Steele et al., 2011). Our study is the first to show that $S$. invicta workers buried food items when their foraging activity was suppressed. The particles relocated on and adjacent to the food may act as physical barriers to block competitors or to block the odors from the food. Interestingly, $S$. invicta workers preferentially transported particles adjacent to the food at the beginning, which 
269

270

271

272

273

274

275

276

277

278

279

280

281

282

283

284

285

286

287

288

may act as "steps" that favor the ants to subsequently move smaller particles onto the food. We also found particles around the food. These particles may be too large to be transported by most of these polymorphic ants, and therefore were left in the midway. Also, some of the small soil particles adjacent to the food were pushed away by the ants when they climbed onto the food, and eventually were distributed around the food.

In this study we did not further investigate whether fire ants return to the hoard food like mammals and birds because: (1) the small piece of food dried within a few days, and therefore was no longer attractive to ants; and (2) the food was provided until colonies were satiated, and it may take a relatively long time when ants are hungry again and therefore may need to return to the hoard food. Someone may argue that $S$. invicta individuals lack the complicated cognitive (memory) processes. However, there is an increasing body of literatures showing the colonylevel cognition in eusocial insects (the internal representations of cognition within the individuals and their interactions among colony members) that can be analogized to the complicated neural cognitive systems in vertebrates (Marshall \& Franks, 2009; Trianni et al., 2011; Sasaki \& Pratt, 2018). For example, Langridge, Franks and Sendova-Franks (2004) found that the emigrating time decreased after Leptothorax albipennis (Curtis) colonies gained experiments from successive emigration events. This study showed that ant colonies can fulfil complicated tasks and improve their collective performance based on memory-like processes. Further studies are needed to verify if the collective decision process could help fire ants to recover the hoard (buried food) like birds and mammals. 
study showed that only a few $S$. invicta workers were particle transporters during the food

burying, and they usually transported particles repeatedly indicating that the burying task may be

carried out by certain groups of workers as an example of behavioral polyethism. Also, our study

showed that the short- and long-distance transport can be performed by the same transporter. It is

likely that the burying behavior requires a simple procedure and no subtask is needed. Previous

studies revealed that ant colony can contain a large proportion of inactive workers (e.g.,

Charbonneau and Dornhaus 2015). Some recent research shows that these inactive individuals

actually had important biological functions (Hasegawa et al. 2016). For example, Charbonneau,

Sasaki and Dornhaus (2017) reported that removal of the active laborers of Temnothorax

rugatulus (Emery) did not decrease the activity level of the whole colony because the inactive

individuals "form a 'reserve' labor force that becomes active when needed." Under the fed

conditions, a large proportion of $S$. invicta workers wandered onto and around on the graph paper.

Apparently, these ants were not involved in particle transport and foraging directly, but they may

act as a pool of "reserve" particle transporters.

The guarding behavior (ants stayed or slowly moved around the food) was commonly

observed in S. invicta when the food was actively foraged (Wang et al., 2016). These guarding

ants may protect the food from competitors before the food was transported back to nests; they

may also act as sentries which protect against parasitoids of the ants when present (Elizalde \&

Folgarait, 2012; Czaczkes, Vellet-Neto \& Ratnieks, 2013). In this study, however, similar 
310 foraging behaviors were suppressed when ants were fed until satiation, and therefore there is no

311 need to guard the food.

312 In this study, we only compared the burying behavior of $S$. invicta when they were starved or fed. It is possible that the burying behavior might be not only caused by the fed conditions, but also triggered by other factors that would suppress the foraging activities. For example, Qin et al. (2017) found that $S$. invicta workers tended to actively transport soil particles onto the food that was previously treated with the solution of sodium dehydroacetate, a repellent against ants.

However, untreated food was actively foraged by ants and few burying behaviors were observed in that study. This indicates that the burying behavior is relatively plastic in its utility. plastic membrane) as the false food (control) to resemble image of the sausage. Creating an appropriate control for this experiment is challenging. It is possible that the wet surface of sausage may also trigger the soil particle transport behaviors of fire ants (Barber et al. 1989, Wang et al. 2018), hence making our control inappropriate. A small, moistened sponge with no nutritional properties may be a more appropriate control to investigate the behavioral patterns of fire ants in response to non-food objects. However, the presence of the sponge may induce the ants to clip it up into soil-sized pieces and potentially use it for nest material further complicating the study.

Broadcast of granular baits is one of the main methods to suppress fire ant populations (e.g., Wylie et al. 2016). Such treatments may need to use large amounts of baits which could deteriorate soon if they are not efficiently foraged by fire ants. Based on our results, we suggest 
331 setting the monitoring stations in fire ant infested areas, and when ants were observed to bury the

332 food (e. g., sausage), massive spreading of the baits should be stopped to reduce the cost and

333 chemical toxicants released into the environments.

334

335

\section{CONCLUSIONS}

In summary, S. invicta workers tend to bury the food items when foraging behaviors were

suppressed (food was provided until satiation in the lab), whereas significantly less soil particles

were transported when ants were starved. In addition, ants preferentially relocated soil particles

adjacent to (in contact with) the food from the beginning, and moved significantly smaller

particles onto the food later. Interestingly, only a small proportion of ants in the colony are

involved with food burying, and those ants tended to transport particles repeatedly, which may

indicate the division of labor during the food burying processes. Food burying behaviors are

usually observed in mammals and birds, and our study is the first to show such behaviors in ants.

Further studies are needed to investigate the ecological and evolutional significance of food

burying behaviors in S. invicta.

\section{Acknowledgments}

348 We thank Hongpeng Xiong, Qinxi Xie, and Rachel Strecker for valuable help in experimental setup and manuscript preparation. We also thank the editor and two reviewers for their constructive comments on this paper. 


\section{References}

353 Abbott HG, Quink TF. 1970. Ecology of eastern white pine seed caches made by small forest mammals. Ecology 51: 271-278 DOI: 10.2307/1933663.

Aitchison J. 1986. The statistical analysis of compositional data. Chapman \& Hall. London.

Ascunce MS, Yang CC, Oakey J, Calcaterra L, Wu WJ, Shih CJ, Goudet J, Ross KG, Shoemaker D. 2011. Global invasion history of the fire ant Solenopsis invicta. Science 331: 1066-1068 DOI: $10.1126 /$ science. 1198734 .

Barber JT, Ellgaard EG, Thien LB, Stack AE. 1989. The use of tools for food transportation by

Beshers SN, Fewell JH. 2001. Models of division of labor in social insects. Annual Review of Entomology 46: 413-440 DOI: 10.1146/annurev.ento.46.1.413.

Charbonneau D, Dornhaus A. 2015. Workers 'specialized' on inactivity: behavioral consistency of inactive workers and their role in task allocation. Behavioral Ecology and Sociobiology 69: 1459-1472. DOI: 10.1007/s00265-015-1958-1.

Charbonneau D, Sasaki T, Dornhaus A. 2017. Who needs 'lazy' workers? Inactive workers act as a 'reserve' labor force replacing active workers, but inactive workers are not replaced when they are removed. PloS One. 12: e0184074 DOI: 10.1371/journal.pone.0184074.

Cook, SC, Eubanks MD, Gold RE, Behmer ST. 2010. Colony-level macronutrient regulation in ants: mechanisms, hoarding and associated costs. Animal Behaviour 79: 429-437 DOI: 10.1016/j.anbehav.2009.11.022. 
373 Czaczkes TJ, Vollet-Neto A, Ratnieks FLW. 2013. Prey escorting behavior and possible

convergent evolution of foraging recruitment mechanisms in an invasive ant. Behavioral Ecology 24: 1177-1184 DOI: 10.1093/beheco/art046.

Darracq AK, Smith LL, Oi DH, Conner LM, McCleery RA. 2017. Invasive ants influence native lizard populations. Ecosphere, 8: e01657 DOI: 10.1002/ecs2.1657.

Elizalde L, Folgarait PJ. 2012. Behavioral strategies of phorid parasitoids and responses of their hosts, the leaf-cutting ants. Journal of Insect Science 12: 135. DOI: 10.1673/031.012.13501.

Frehner EH, Buechley ER, Christensen T, Şekercioğlu ÇH. 2017. Subterranean caching of domestic cow (Bos taurus) carcasses by American badgers (Taxidea taxus) in the Great Basin Desert, Utah. Western North American Naturalist 77: 124-129 DOI: 10.3398/064.077.0114.

Greenhouse SW, Geisser W. 1959. On methods in the analysis of profile data. Psychometrika 24: 95-112.

Hasegawa E, Ishii Y, Tada K, Kobayashi K, Yoshimura J. 2016. Lazy workers are necessary for long-term sustainability in insect societies. Scientific Reports 6: 20846. DOI: $10.1038 /$ srep20846.

Hayashida M. 1989. Seed dispersal by red squirrels and subsequent establishment of Korean pine. Forest Ecology and Management 28: 115-129 DOI: 10.1016/0378-1127(89)90064-9.

Hölldobler B, Wilson EO. (1990). The Ants. Harvard/Belknap.

Holway DA, Lach L, Suarez AV, Tsutsui ND, Case TJ. 2002. The causes and consequences of ant invasions. Annual Review of Ecology Evolution and Systematics 33: 181-233 DOI: 
10.1146/annurev.ecolsys.33.010802.150444.

395

396

397

398

399

400

401

402

403

404

405

406

Kucera M, Malmgren BA. 1998. Logratio transformation of compositional data: a resolution of the constant sum constraint. Marine Micropaleontology 34: 117-120 DOI: 10.1016/S03778398(97)00047-9.

Langridge EA, Franks NR, Sendova-Franks AB. 2004. Improvement in collective performance with experience in ants. Behavioral Ecology and Sociobiology 56: 523-529 DOI 10.1007/s00265-004-0824-3.

Maák I, Lörinczi G, Le Quinquis P, Módra G, Bovet D, Call J, d'Ettorre P. 2017. Tool selection during foraging in two species of funnel ants. Animal Behaviour 123: 207-216 DOI: 10.1016/j.anbehav.2016.11.005.

Marshall JAR, Franks NR. 2009. Colony-level cognition. Current Biology 19: R395-R396 DOI: 10.1016/j.cub.2009.03.011.

Miyaki M. 1987. Seed dispersal of the Korean pine, Pinus koraiensis, by the red squirrel, Sciurus vulgaris. Ecological Research 2: 147-157 DOI: 10.1007/BF02346923.

Morrill WL. 1972. Tool using behavior of Pogonomyrmex badius (Hymenoptera: Formicidae). Florida Entomologist 55: 59-60 DOI: 10.2307/3493647.

Morrison LW, Porter SD, Daniels E, Korzukhin MD. 2004. Potential global range expansion of the invasive fire ant, Solenopsis invicta. Biological Invasions 6: 183-191 DOI: 10.1023/B:BINV.0000022135.96042.90.

O’brien KS, Hooper-Bùi LM. 2005. Hunger in red imported fire ants and their behavioral response to two liquid bait products. Journal of Economic Entomology 98: 2153-2159 DOI: 
10.1093/jee/98.6.2153.

416

417

418

419

420

421

422

423

424

425

426

427

428

429

430

431

432

433

434

435

Orrock JL, Danielson BJ. 2004. Rodents balancing a variety of risks: invasive fire ants and indirect and direct indicators of predation risk. Oecologia 140: 662-667 DOI: 10.1007/s00442-004-1613-4.

Qin WQ, Xiong HP, Wen YZ, Wen XJ, Wang H, Fang YH, Ma T, Sun ZH, Chen XY, Wang C. 2017. Laboratory and field evaluation of the repellency of six preservatives to red imported fire ants (Hymenoptera: Formicidae). Journal of Asia-Pacific Entomology 20: 535-540 DOI: 10.1016/j.aspen.2017.03.011.

Rust MK, Su NY. 2012. Managing social insects of urban importance. Annual Review of Entomology 57: 355-375 DOI: 10.1146/annurev-ento-120710-100634.

Sasaki T, Pratt SC. 2018. The Psychology of superorganisms: Collective decision-making by insect societies. Annual Review of Entomology 63: 259-275 DOI: 10.1146/annurev-ento020117-043249.

Steele M. A, Bugdal M, Yuan A, Bartlow A, Buzalewski J, Lichti N, Swihart R. 2011. Cache placement, pilfering, and a recovery advantage in a seed-dispersing rodent: could predation of scatter hoarders contribute to seedling establishment? Acta Oecologica 37: 554-560 DOI: 10.1016/j.actao.2011.05.002.

Su HJ, Ma JZ, Zong C. 2007. Behavior comparison between four diurnal animals on seed consumption and hoarding of Korean Pine. Chinese Journal of Zoology 42: 10-16 DOI: 10.13859/j.cjz.2007.02.003.

Thawley CJ, Langkilde T. 2016. Invasive fire ant (Solenopsis invicta) predation of eastern fence 
lizard (Sceloporus undulatus) eggs. Journal of Herpetology 50: 284-288 DOI: 10.1670/15017.

Trianni V, Tuci E, Passino KM, Marshall JA. 2011. Swarm cognition: an interdisciplinary approach to the study of self-organising biological collectives. Swarm Intelligence 5: 3-18 DOI: $10.1007 / \mathrm{s} 11721-010-0050-8$.

Tschinkel WR, Rink WJ, Kwapich CL. 2015. Sequential subterranean transport of excavated sand and foraged seeds in nests of the harvester ant, Pogonomyrmex badius. PloS One 10: e0139922 DOI: 10.1371/journal.pone.0139922.

Tschinkel, W. R. (2011). The organization of foraging in the fire ant, Solenopsis invicta. Journal of Insect Science 11: 26 DOI: 10.1673/031.011.0126.

Wang C, Chen X, Hooper-Bùi LM, Strecker R, Wen YZ, Qin WQ, Ma T, Sun ZH, Chen XY, Wen XJ. 2018. How do groups of red imported fire ants (Hymenoptera: Formicidae) feed on a droplet of sugar water? Insect Science 25: 499-507 DOI: 10.1111/1744-7917.12435.

Wang C, Chen X, Strecker R, Henderson G, Wen XJ, Hooper-Bùi LM. 2016. Individual and cooperative food transport of the red imported fire ant (Hymenoptera: Formicidae): Laboratory observations. Journal of Insect Behavior 29: 99-107 DOI: 10.1007/s10905016-9546-4.

Wauters LA, Tosi G, Gurnell J. 2002. Interspecific competition in tree squirrels: do introduced grey squirrels (Sciurus carolinensis) deplete tree seeds hoarded by red squirrels (S. vulgaris)? Behavioral Ecology and Sociobiology 51: 360-367 DOI: 10.1007/s00265-001-0446-y. Wetterer JK. 2013. Exotic spread of Solenopsis invicta Buren (Hymenoptera: Formicidae) 
457

458

459

460

461

462

463

464

465

466

467

468

469

470

471

472

473

474

475

476

477

beyond North America. Sociobiology 60: 50-55 DOI: 10.13102/sociobiology.v60i1.50-55.

Wilder SM, Holway DA, Suarez AV, LeBrun EG, Eubanks MD. 2011. Intercontinental

differences in resource use reveal the importance of mutualisms in fire ant invasions.

Proceedings of the National Academy of Sciences 108: 20639-20644 DOI:

10.1073/pnas. 1115263108 .

Williams DF, Collins HL, Oi DH. 2001. The red imported fire ant (Hymenoptera: Formicidae): an historical perspective of treatment programs and the development of chemical baits for control. American Entomologist 47: 146-159 DOI: 10.1093/ae/47.3.146.

Wylie FR, Janssen-May S. 2017. Red imported fire ant in Australia: What if we lose the war? Ecological Management \& Restoration 18: 32-44 DOI: 10.1111/emr.12238.

Wylie R, Jennings C, McNaught MK, Oakey J, Harris EJ. 2016. Eradication of two incursions of the red imported fire ant in Queensland, Australia. Ecological Management \& Restoration. 17: 22-32 DOI: 10.1111/emr.12197.

Xu YJ, Lu YY, Zeng L, Liang GW. 2007. Foraging behavior and recruitment of red imported fire ant Solenopsis invicta Buren in typical habitats of South China. Acta Ecologica Sinica 27: 855-860 DOI: 10.3321/j.issn:1000-0933.2007.03.004.

Zeng L, Lu YY, He XF, Zhang WQ, Liang GW. 2005. Identification of red imported fire ant, Solenopsis invicta, to invade mainland China and infestation in Wuchuan, Guangdong. Chinese Bulletin of Entomology 42: 144-148 DOI: 10.3969/j.issn.0452-8255.2005.02.007.

Zong C, Mei S, Santicchia F, Wauters LA, Preatoni DG, Martinoli A. 2014. Habitat effects on hoarding plasticity in the Eurasian red squirrel (Sciurus vulgaris). Hystrix, the Italian 
Journal of Mammalogy. 25: 14-17 DOI: 10.4404/hystrix-25.1-10160.

Zong C, Wauters LA, Van Dongen S, Mari V, Romeo C, Martinoli A, Preatoni D. Tosi G. 2010. Annual variation in predation and dispersal of Arolla pine (Pinus cembra L.) seeds by Eurasian red squirrels and other seed-eaters. Forest Ecology and Management 260: 587-594 DOI: $10.1016 /$ j.foreco.2010.05.014.

\section{Figure Legends}

Fig. 1: (a) Experimental arenas, and (b) diagram depicting the experimental design. Solenopsis invicta colonies were collected from the field by transferring the mound materials containing large number of eggs, larvae, pupae and adults to a plastic box (nest box), which was then connected with a foraging area where the experiments were set-up. The first foraging test was conducted under starved conditions, and the second foraging test and videotaping were conducted when food was provided until satiation of the colonies. The photograph was taken by the first author.

Fig. 2: Examples of behaviors of Solenopsis invicta workers in response to the real food (small pieces of sausage) and false food (square acrylic plates) when they were starved or fed until satiation. Under starved conditions, sausage attracted a large number of foraging ants to dissect and transport the food, but few food-burying behaviors were observed. However, when ants were fed until satiation they tended to bury the sausage with soil particles instead of transporting the 
499

500

501

502

503

504

505

506

507

508

509

510

511

512

513

514

515

516

517

food. The false food caused few food-transport or burying activities whenever the ants were starved or fed. The photographs were taken by the first author.

Fig. 3: (a) Percentage of replicates that showed active food-transport behaviors, (b) number of foraging ants (mean $\pm \mathrm{SE}$ ) on and adjacent to the food, (c) percentage of replicates that showed active food-burying behaviors, and (d) weight of soil particles (mean $\pm \mathrm{SE}$ ) relocated on the food (real or false) and graph paper are presented. Number of foraging ants and weight of particles were compared using the two-way ANOVA with ant colony as a random factor and treatment as a fixed factor. Different letters indicate significant differences $(P<0.05)$.

Fig. 4: (a) Screenshots showing that soil particles were first relocated adjacent to the food (as shown at $15 \mathrm{~min}$ ), and then transported onto the food (as shown at $60 \mathrm{~min}$ ). Meanwhile, particles were relocated around the food (as shown at 60 and $120 \mathrm{~min}$ ). The video was taken by the first author. (b) At each time interval, percentage (mean $\pm \mathrm{SE}$ ) of particles on, adjacent to, or around the food was transformed to $\ln$-ratios (the untransformed data were presented here). The transformed data were then compared using repeated measures ANOVA with time as the withinsubjects effect and location as the between-subjects effect, and one-way ANOVA was performed for each time interval for multiple comparisons. Different letters indicate significant differences at each time interval $(P<0.05)$. (c) The size distribution of particles that were adjacent to $(\mathrm{n}=$ $148)$, on $(n=165)$, or around $(n=307)$ the food at the end of the experiment. 
520 Fig. 5: (a) Number of Solenopsis invicta workers that exhibited wandering, foraging and food-

521 burying activities was compared using repeated measures ANOVA with time as the within-

522 subjects effect and behavior as the between-subjects effect, and one-way ANOVA was

523 performed for each time interval for multiple comparisons. Different letters indicate significant

524 differences at each time interval $(P<0.05)$. (b) The percentage of large, medium and small

525 workers that exhibited the food-burying behavior. In total, 133 soil transporters were recorded

526 from the 10 videos. (c) Time (mean \pm SE) spent repeatedly transporting the particles by each ant,

(d) number (mean $\pm \mathrm{SE}$ ) of relocated particles for each ant, and (e) the average time (mean $\pm \mathrm{SE}$ )

spent transporting each particle by each ant were compared among ant sizes (large, medium and small workers) using the one-way ANOVA. Different letters indicate significant differences $(P<$ $0.05)$.

Fig. 6: (a) Examples of trails for the transport of soil particles by Solenopsis invicta workers. The hollow circles indicate the location that the particles were placed. Based on the location where the soil particle was picked and placed on the food or graph paper, 12 patterns were identified and indicated by different colors. (b) Frequency of each pattern of particle transport by ants. In total, 881 transport events were recorded and classified. (c) Time (mean \pm SE) spent for each transport event was compared among transport patterns using the one-way ANOVA. Different letters indicate significant differences $(P<0.05)$. 
540 Fig. 7: An example of the field observation, in which the active foraging behaviors were

541 observed. No soil particle was found on or adjacent to the food, but some particles were found

542 around the food. The photograph was taken by the first author. 


\section{Figure 1}

Experimental arenas and diagram depicting the experimental design

(a) Experimental arenas, and (b) diagram depicting the experimental design. Solenopsis

invicta colonies were collected from the field by transferring the mound materials containing large number of eggs, larvae, pupae and adults to a plastic box (nest box), which was then connected with a foraging area where the experiments were set-up. The first foraging test was conducted under starved conditions, and the second foraging test and videotaping were conducted when food was provided until satiation of the colonies. The photograph was taken by the first author. 


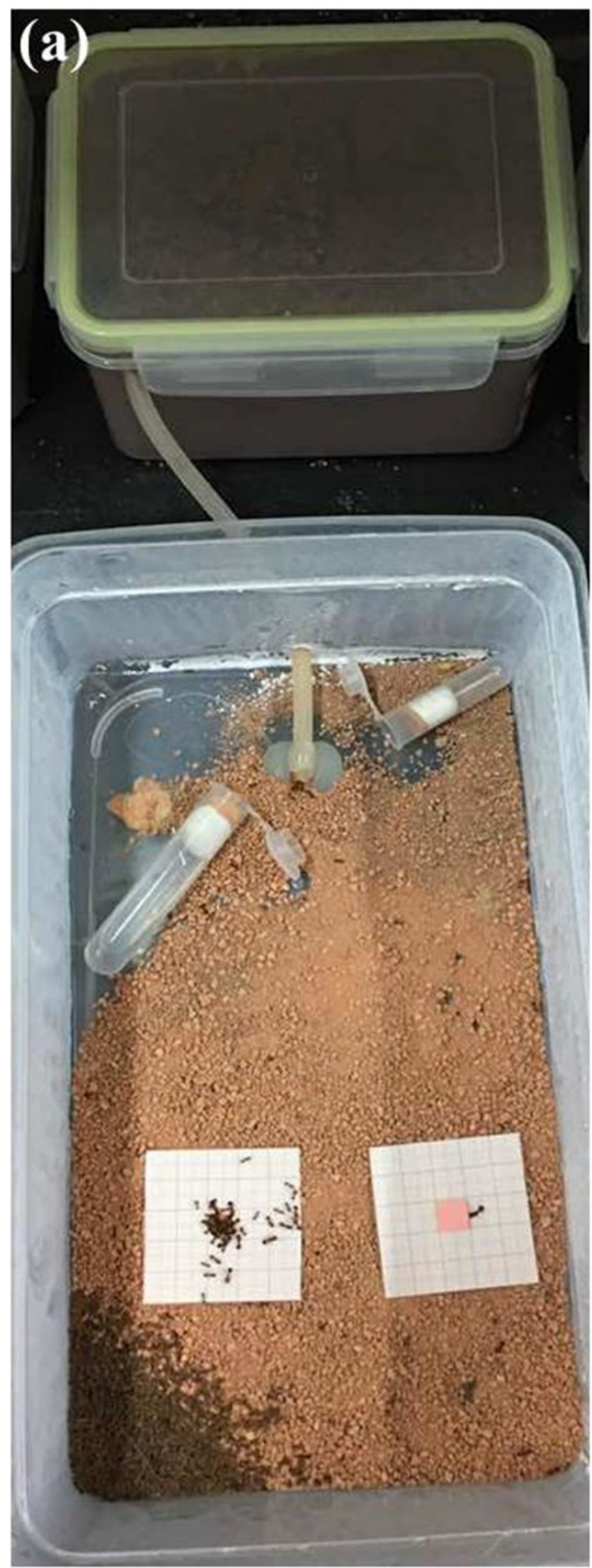

(b)

\section{Ant collection}

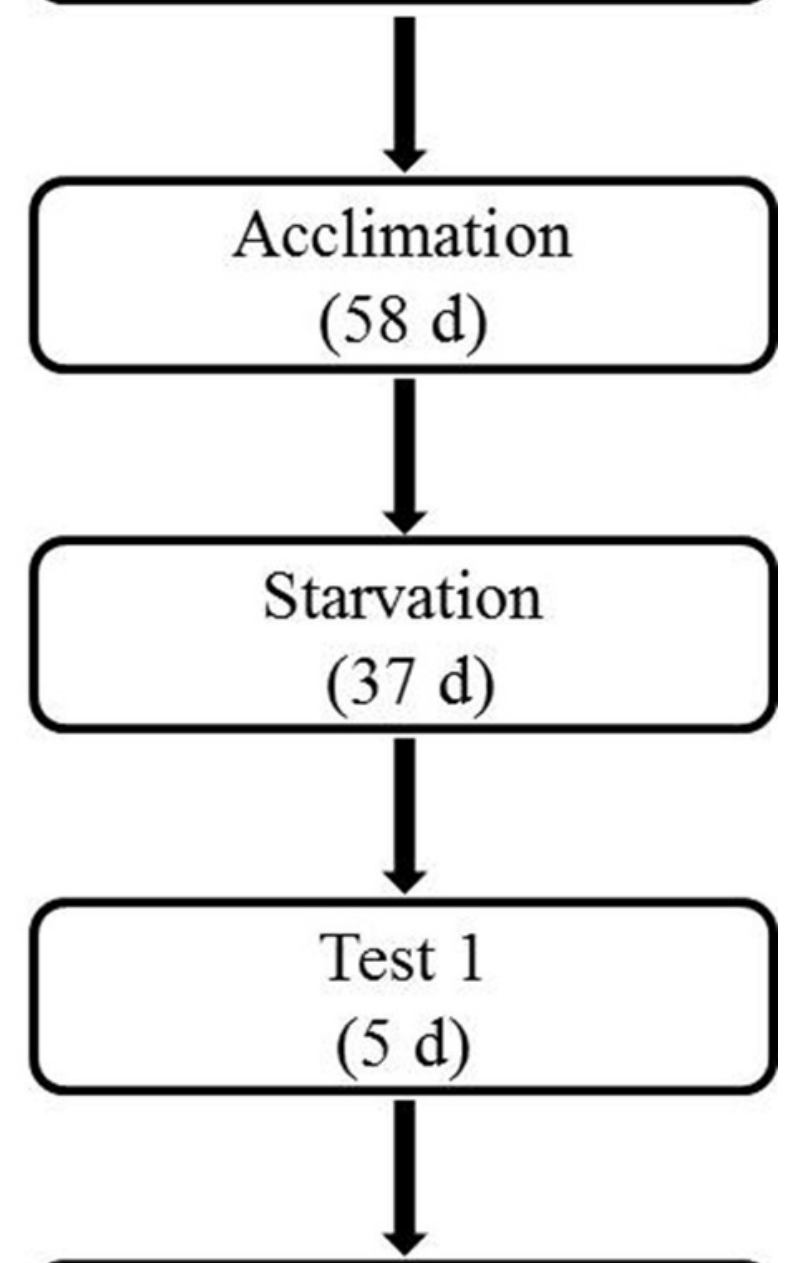

Fed to satiation

(15 d)

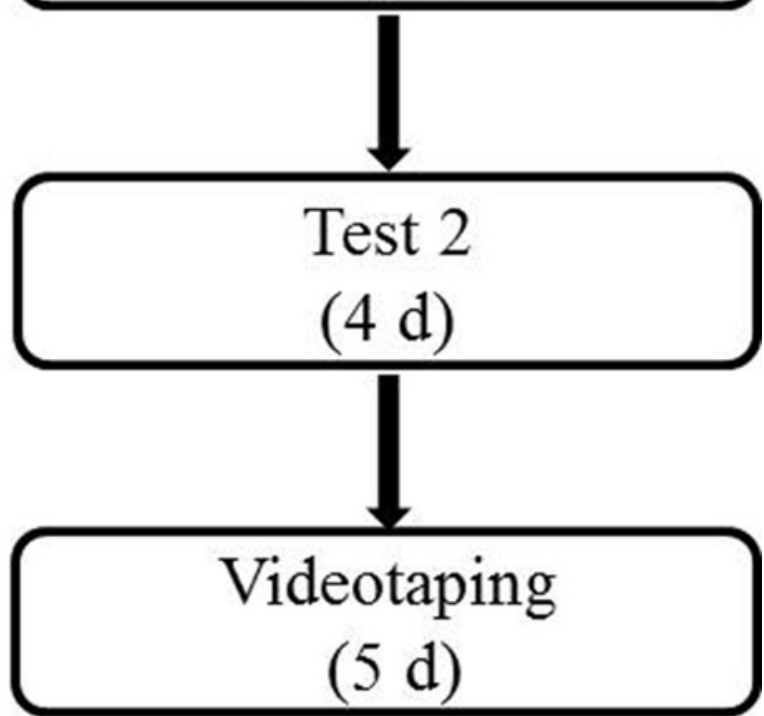




\section{Figure 2}

Examples of food-transport and food-burying behaviors of Solenopsis invicta workers

Examples of behaviors of Solenopsis invicta workers in response to the real food (small pieces of sausage) and false food (square acrylic plates) when they were starved or fed until satiation. Under starved conditions, sausage attracted a large number of foraging ants to dissect and transport the food, but few food-burying behaviors were observed. However, when ants were fed until satiation they tended to bury the sausage with soil particles instead of transporting the food. The false food caused few food-transport or burying activities whenever the ants were starved or fed. The photographs were taken by the first author. 


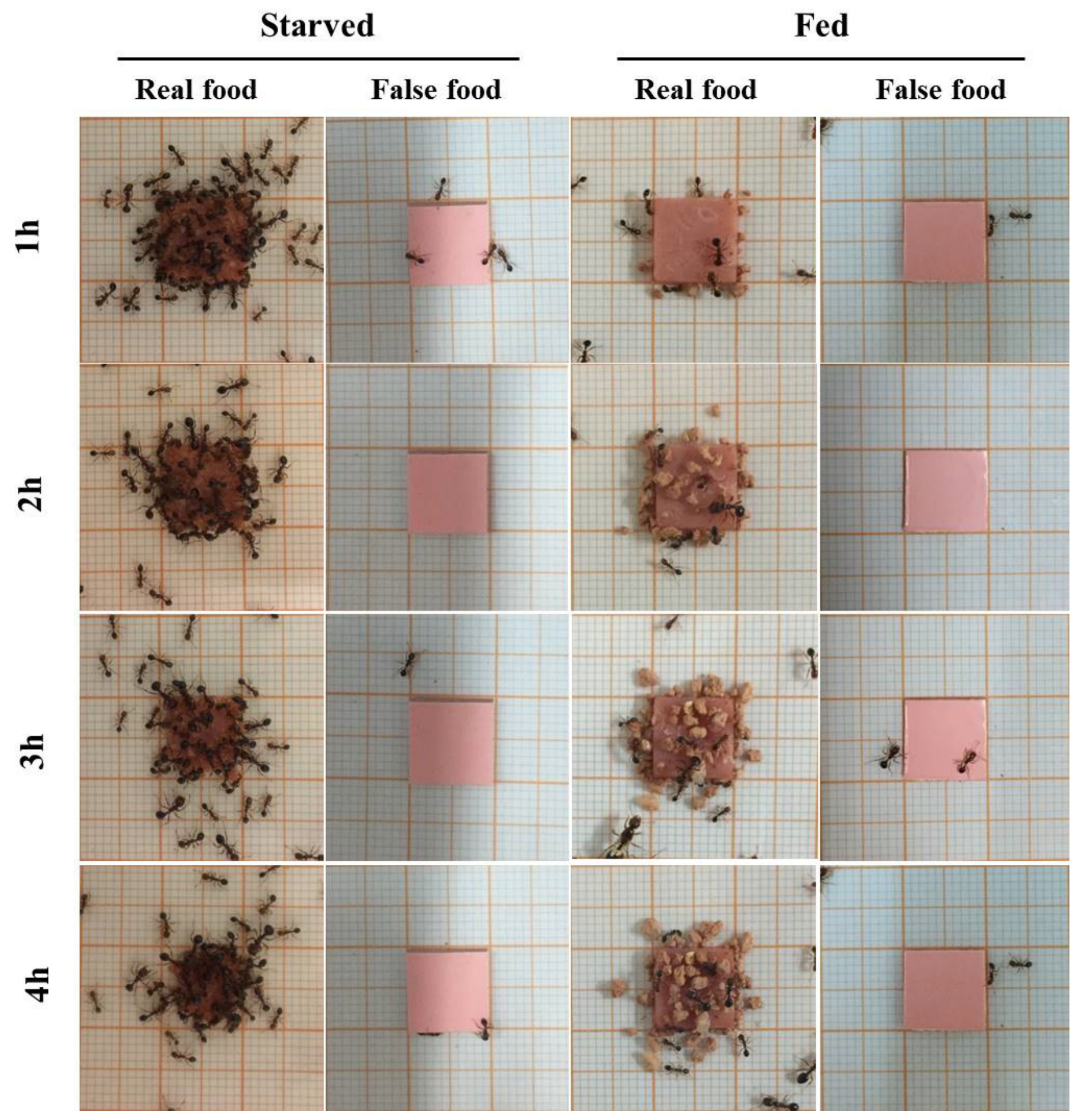


Figure 3

Food-transport and food-burying behaviors

(a) Percentage of replicates that showed active food-transport behaviors, (b) number of foraging ants (mean \pm SE ) on and adjacent to the food, (c) percentage of replicates that showed active food-burying behaviors, and (d) weight of soil particles (mean \pm SE ) relocated on the food (real or false) and graph paper are presented. Number of foraging ants and weight of particles were compared using the two-way ANOVA with ant colony as a random factor and treatment as a fixed factor. Different letters indicate significant differences $(P<$ 0.05).
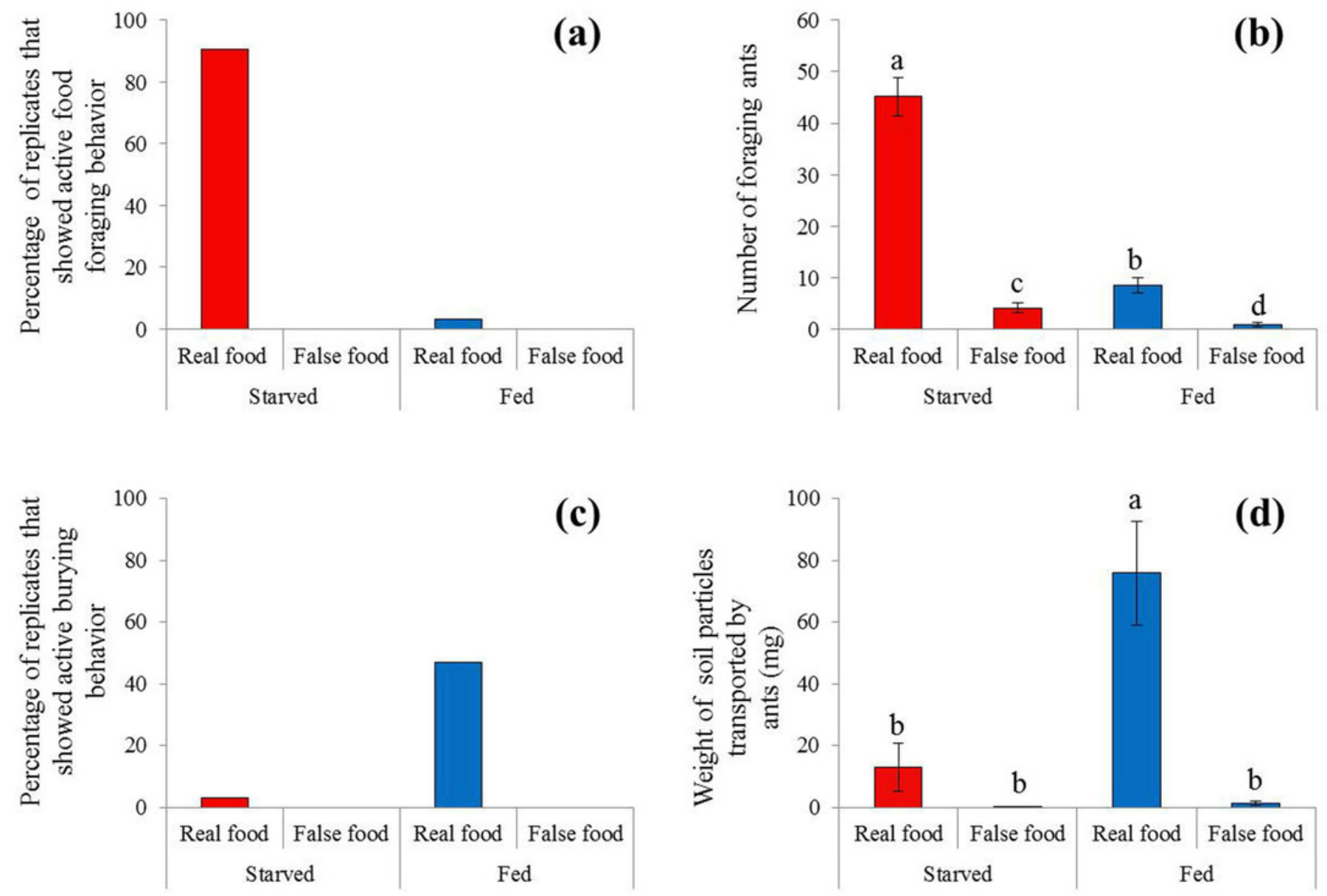


\section{Figure 4}

Soil particles relocated on, adjacent to, or around the food

(a) Screenshots showing that soil particles were first relocated adjacent to the food (as shown at $15 \mathrm{~min}$ ), and then transported onto the food (as shown at $60 \mathrm{~min}$ ). Meanwhile, particles were relocated around the food (as shown at 60 and $120 \mathrm{~min}$ ). The video was taken

by the first author. (b) At each time interval, percentage (mean $\pm \mathrm{SE}$ ) of particles on, adjacent to, or around the food was transformed to In-ratios (the untransformed data were presented here). The transformed data were then compared using repeated measures ANOVA with time as the within-subjects effect and location as the between-subjects effect, and one-way ANOVA was performed for each time interval for multiple comparisons. Different letters indicate significant differences at each time interval $(P<0.05)$. (c) The size distribution of particles that were adjacent to $(n=148)$, on $(n=165)$, or around $(n=307)$ the food at the end of the experiment. 


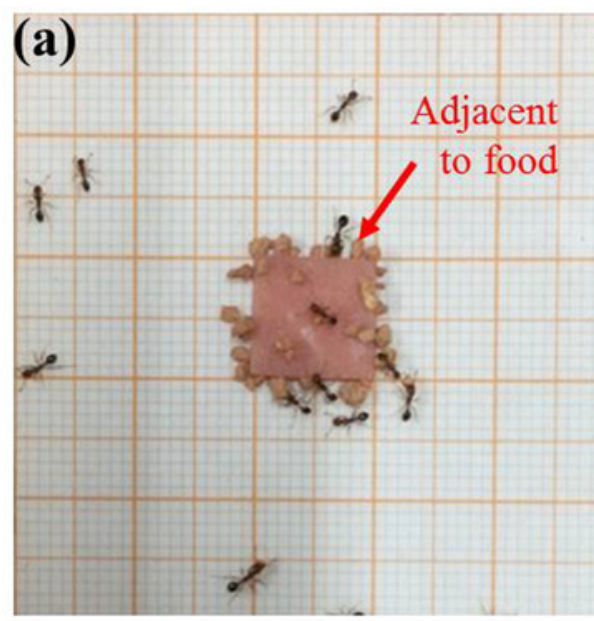

$15 \mathrm{~min}$

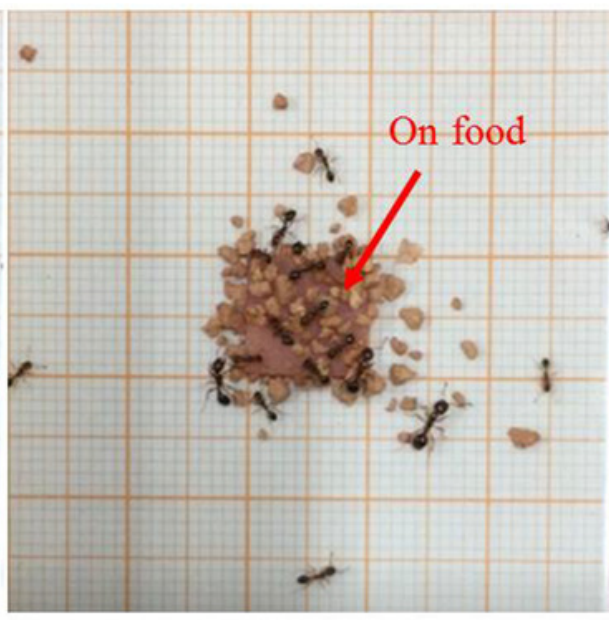

$60 \mathrm{~min}$

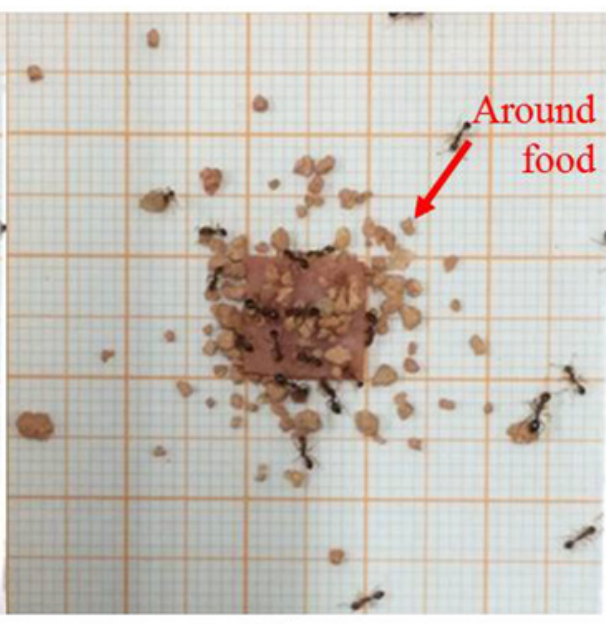

$120 \mathrm{~min}$

(b)

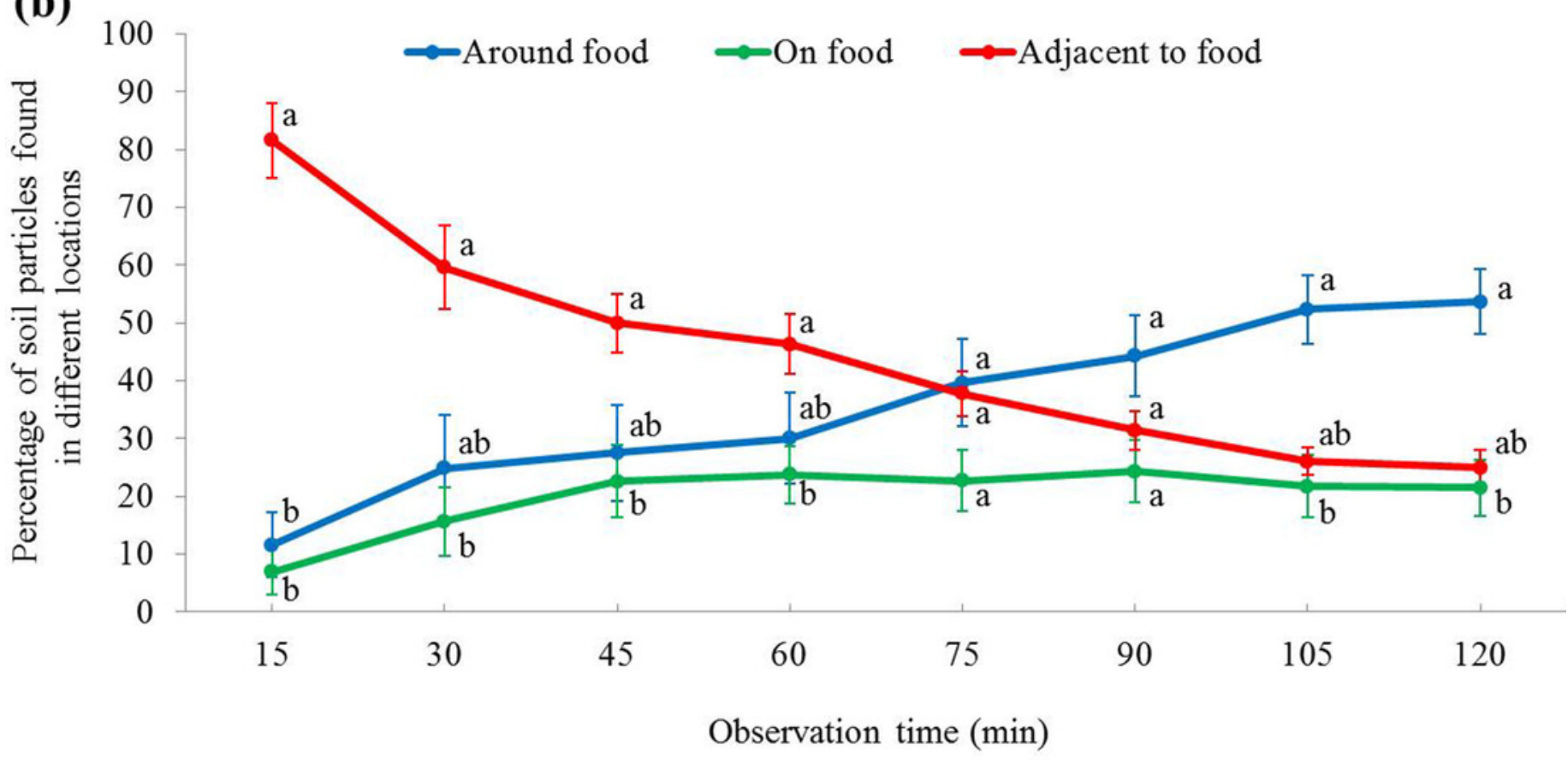

(c)
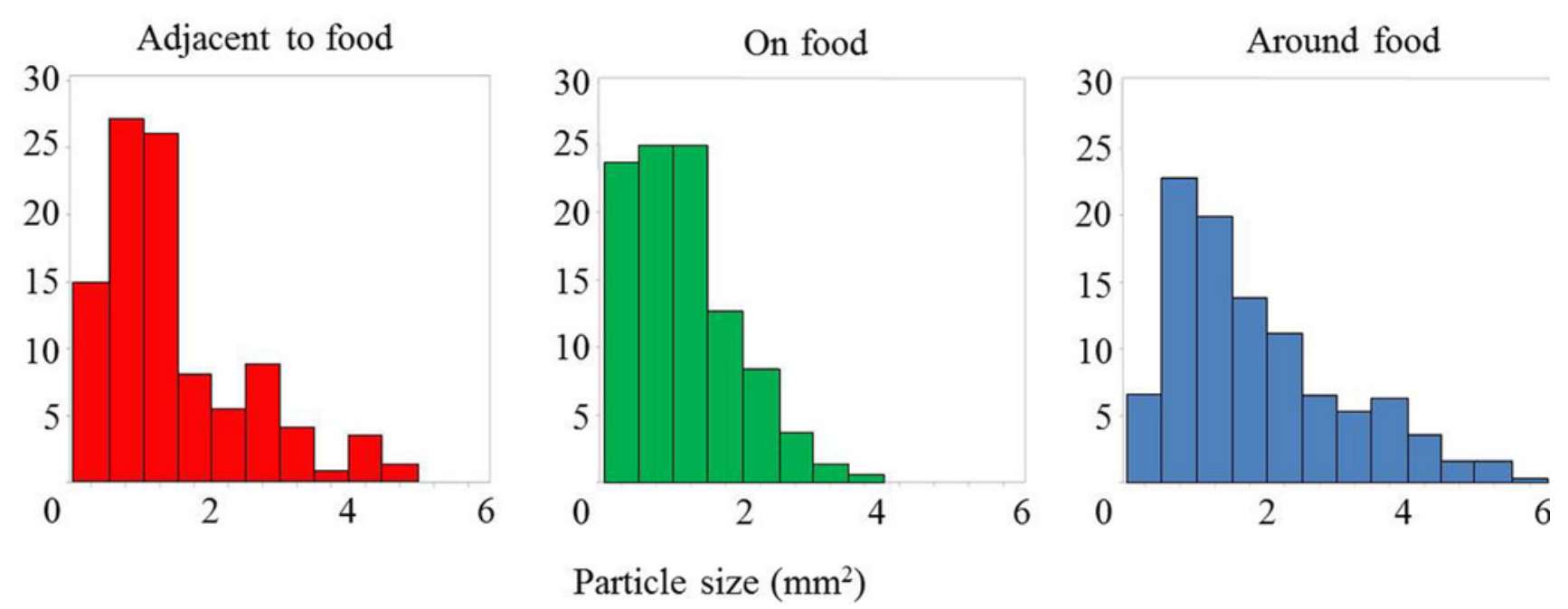


\section{Figure 5}

Behaviors of Solenopsis invicta workers

(a) Number of Solenopsis invicta workers that exhibited wandering, foraging and foodburying activities was compared using repeated measures ANOVA (SPSS 24) with time as the within-subjects effect and behavior as the between-subjects effect, and one-way ANOVA was performed for each time interval for multiple comparisons. Different letters indicate significant differences at each time interval $(P<0.05)$. (b) The percentage of large, medium and small workers that exhibited the food-burying behavior. In total, 133 soil transporters were recorded from the 10 videos. (c) Time (mean \pm SE ) spent repeatedly transporting the particles by each ant, (d) number (mean $\pm \mathrm{SE}$ ) of relocated particles for each ant, and (e) the average time (mean $\pm \mathrm{SE}$ ) spent transporting each particle by each ant were compared among ant sizes (large, medium and small workers) using the one-way ANOVA. Different letters indicate significant differences $(P<0.05)$ 
(a)

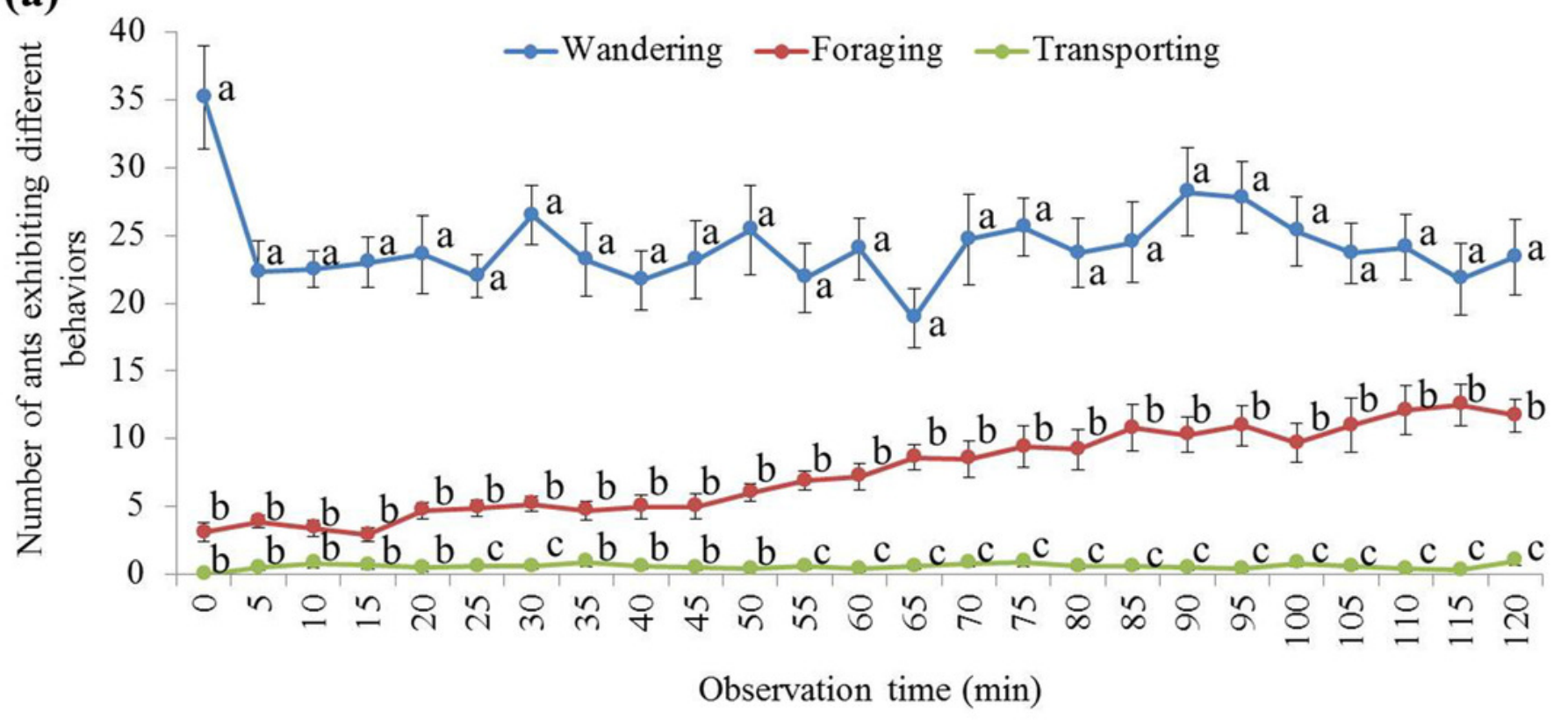

(b)

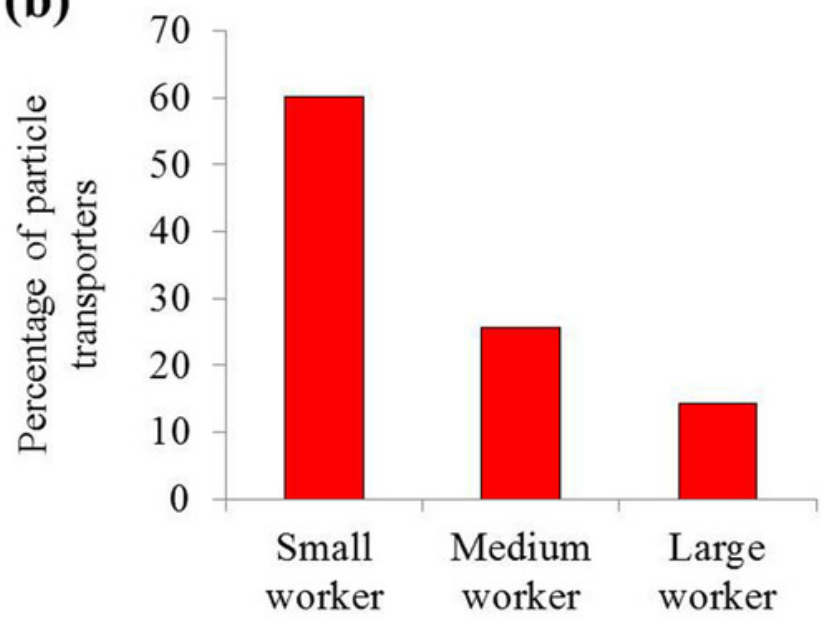

(d)

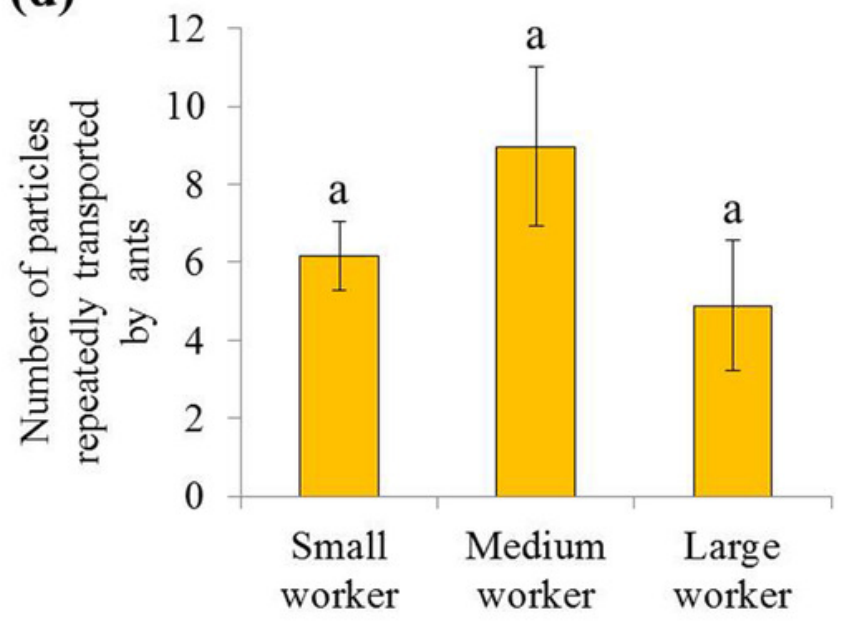

(c)

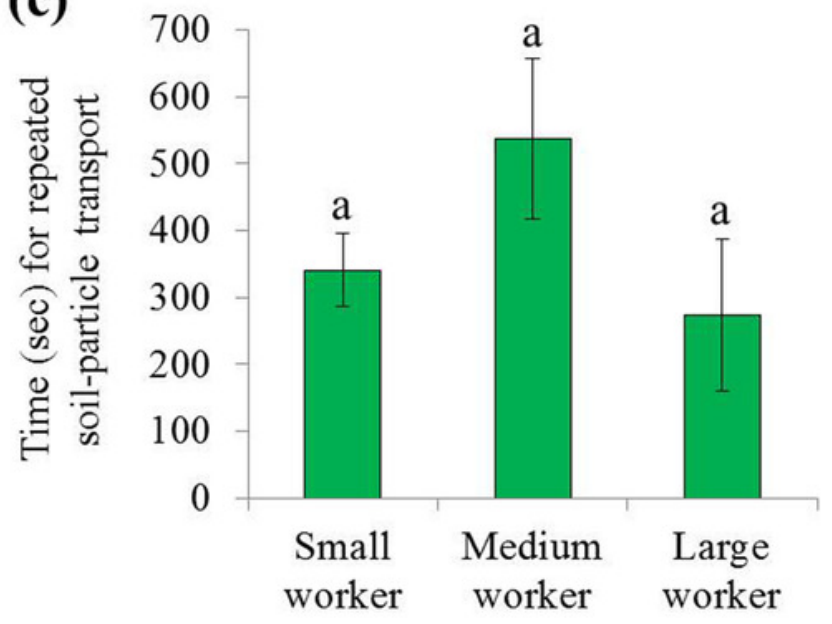

(e)

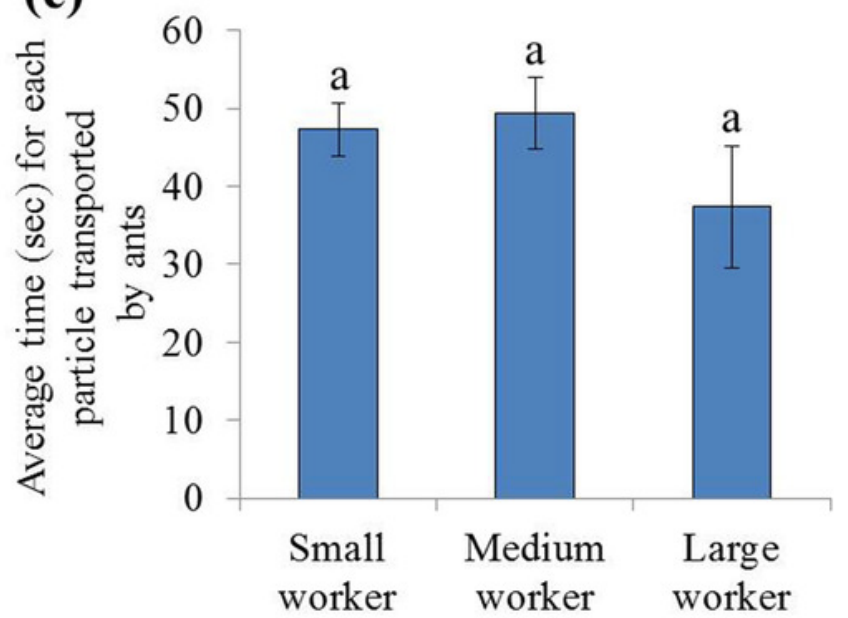




\section{Figure 6}

Patterns of particle transport

(a) Examples of trails for the transport of soil particles by Solenopsis invicta workers. The hollow circles indicate the location that the particles were placed. Based on the location where the soil particle was picked and placed on the food or graph paper, 12 patterns were identified and indicated by different colors. (b) Frequency of each pattern of particle transport by ants. In total, 877 transport events were recorded and classified. (c) Time (mean $\pm \mathrm{SE}$ ) spent for each transport event was compared among transport patterns using the oneway ANOVA. Different letters indicate significant differences $(P<0.05)$. 


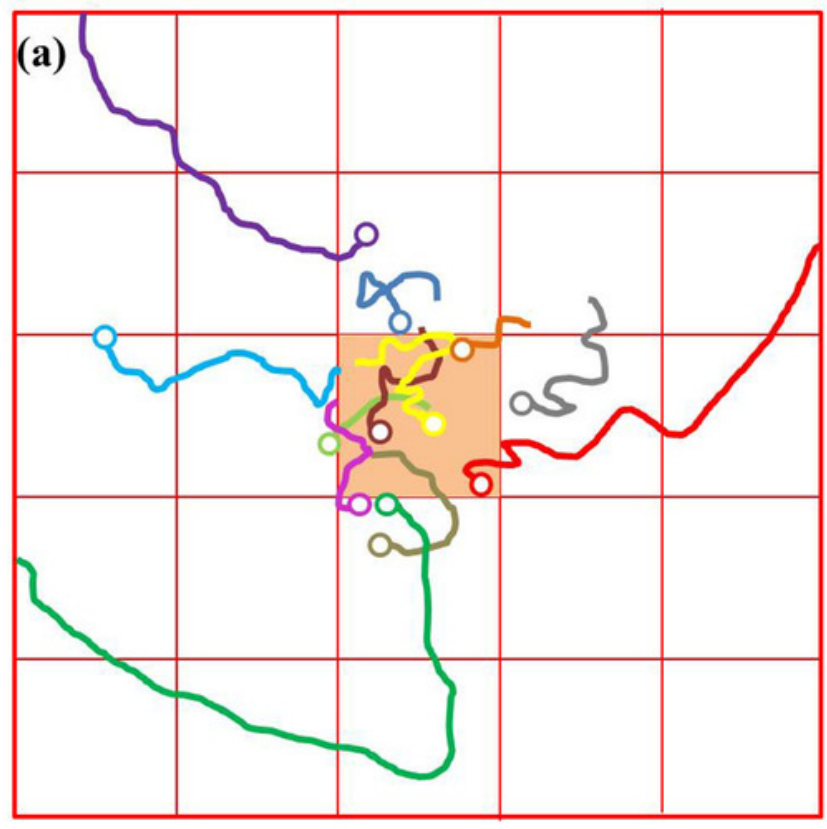

(b)
Long-distance transport:

Ad: The soil particle was picked up from the foraging arena and relocated adjacent to food

On: The soil particle was picked up from the foraging arena and relocated on food

Ar: The soil particle was picked up from the foraging arena and relocated around food

Short-distance transport:

ArAd: The soil particle around food was picked up and placed adjacent to food

ArOn: The soil particle around food was picked up and placed on food

ArAr: The soil particle around food was picked up and again placed around food

OnAd: The soil particle on food was picked up and placed adjacent to food

OnOn: The soil particle on food was picked up and again placed on food

OnAr: The soil particle on food was picked up and placed around food

AdAd: The soil particle adjacent to food was picked up and again placed adjacent to food

AdOn: The soil particle adjacent to food was picked up and placed on food

AdAr: The soil particle adjacent to food was picked up and placed around food (c)

(c) 100

(5)

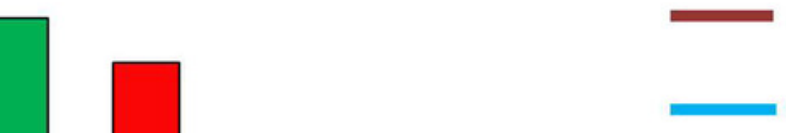




\section{Figure 7}

An example of the field observation

An example of the field observation, in which the active foraging behaviors were observed.

No soil particle was found on or adjacent to the food, but some particles were found around the food. The photograph was taken by the first author. 


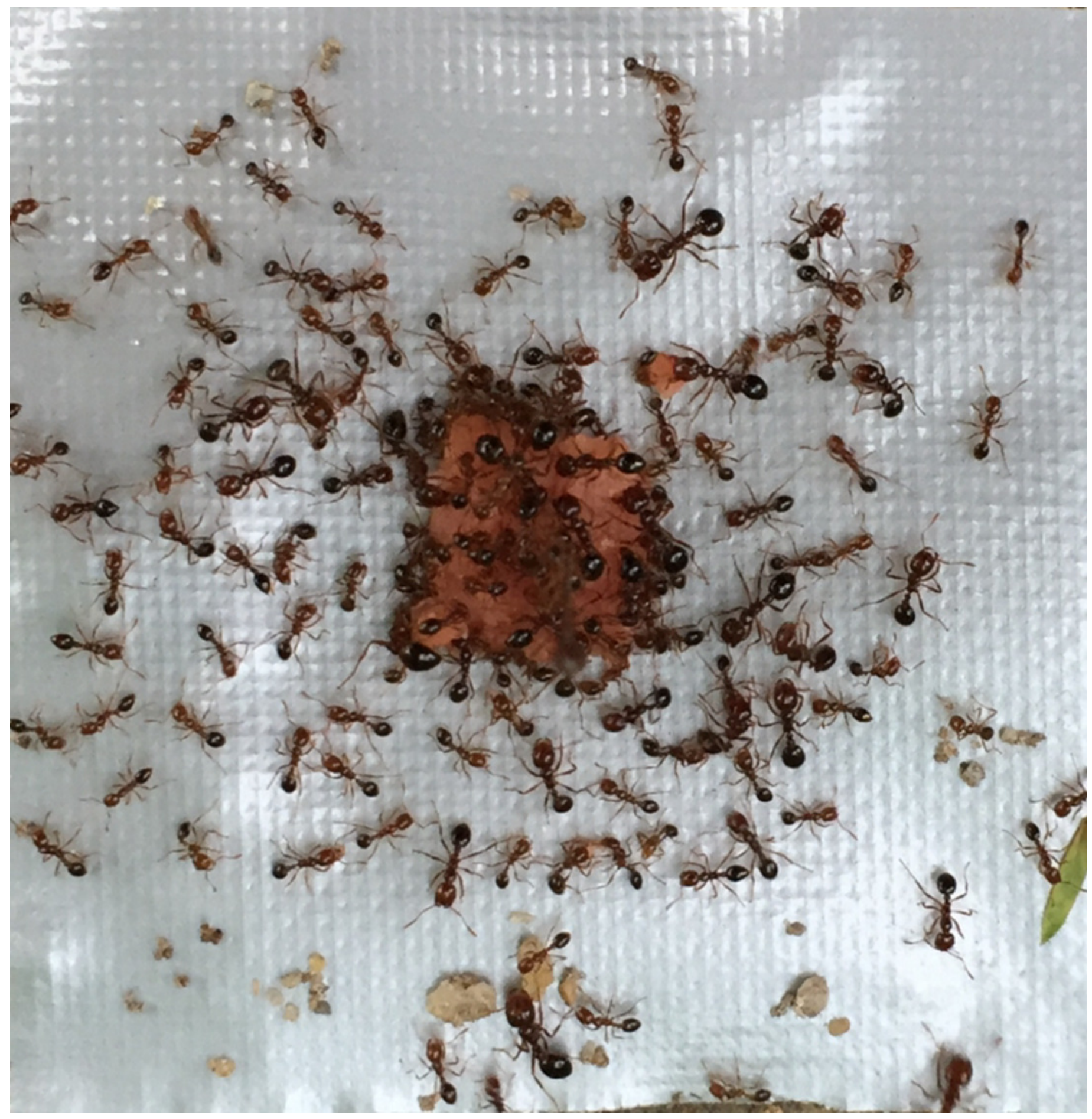

